\title{
VARIACION DE LA PROBABILIDAD DE REFUERZO EN EL SEGUNDO COMPONENTE DE UN PROGRAMA MULTIPLE Y SUS EFECTOS SOBRE EL GRADIENTE DE GENERALIZACION*
}

Se realizarán tres experimentos con el fin de estudiar los efectos de los cambios de la probabilidad de reforzamiento en el segundo componente de un múltiple y las tasas de respuestas así logradas sobre el gradiente de generalización. Los tres sujetos de cada experimento recibirán inicialmente entrenamiento no diferencial en un programa múltiple cuyos estímulos asociados son dos tonos de $1000 \mathrm{cps} .\left(S_{1}\right)$ y $950 \mathrm{cps}$. $\left(S_{2}\right)$. En el Experimento I no habrá variación en la probabilidad de refuerzo. Sin embargo, habrá cambios que permiten esperar un aumento en la tasa. En el Experimento II se incrementará la probabilidad de refuerzo en el segundo componente y la naturaleza del programa utilizado permite esperar también un aumento en la tasa de respuestas. En el Experimento III habrá una disminución en la mencionada probabilidad en el componente 2 y se espera un aumento en la tasa.

Estas expectancias deben entenderse como una línea de base determinada a la cual pretendemos llegar antes de efectuar la prueba de generalización para cada uno de los grupos.

Actualmente se utiliza el término "control del estímulo" para designar una función empírica continua cuyos extremos son la discriminación y la generalización. La utilización de este término evita una serie de confusiones generadas al intentar describir estos fenómenos separadamente. Un ejemplo de estas confusiones es el intento de formular la generalización como una ausencia de discriminación. Especifiquemos dos conceptos que son claves en la afirmación anterior y que por lo general su significado no es lo suficientemente preciso. Estos conceptos son "control del estímulo" y "función empírica continua". La siguiente cita puede definirnos correctamente "control del estímulo": "Si un cambio en algún aspecto de un estímulo que antecede a una respuesta resulta en un cambio en alguna medida de la respuesta, diremos que el estimulo ejerce un control sobre la respuesta" (Eckeaman, 1969). Desafortunadamente no disponemos de una definición similar para "función empírica continua". Este concepto trata de recordar las funciones continuas en matemáticas, sin embargo, no es claro en qué consiste la "continuidad empírica". Tal vez hablar de que no existen restricciones empíricas para graficar gradientes de generalización en un plano cartesiano aclare esta ide de "continuidad empírica".

Dentro de esta gama "continua" de posibles gradientes de generalización existe una subclase de ellos que presentan una cierta "anomalía" que recibe el nombre de desplazamiento de pico. Más explícitamente, se presentan en ocasiones gradientes en los cuales el número mayor de respuestas no se da ante el estímulo discriminativo (desplazamiento de pico positivo) y el número menor de respuestas no se da ante el estímulo delta (desplazamiento de pico negativo). El desplazamiento de pico se da generalmente cuando el entrenamiento en discriminación se hace mediante ciertos programas múltiples. Hay una serie de trabajos que estudian sistemáticamente una de las variables que se manipulan en un programa múltiple: la probabilidad de refuerzo en cada

\footnotetext{
* Esta investigación se lleva a cabo en la Universidad Pedagógica Nacional en el Departamento de Educación.
} 
componente del programa. El caso típico, múltiple IV 1-EXT., podría expresarse de la siguiente manera:

$$
\begin{aligned}
& P_{s 1}(R)=x \text { con } x \# O y \\
& P_{s 2}(R)=O . S_{1} y S_{2}
\end{aligned}
$$

Representan los estímulos asociados con cada componente del múltiple; $P_{\mathrm{S} 1}(\mathrm{R})$ se leería entonces "la probabilidad de que una respuesta $\mathrm{R}$ sea reforzada dado que el estímulo $\mathrm{S} 1$ está presente. Cuando $\mathrm{P}_{\mathrm{s} 2}(\mathrm{R})=\mathrm{O}$, S2 sería el típico estímulo Delta; sin embargo es concebible que $P_{S 2}(R)=y$ con $0 \leq y \leq 1$. La serie de trabajos a la cual nos hemos referido, hablando de una manera más precisa, se ha encargado de establecer una relación entre el cambio de $P_{\mathrm{S} 2}(R)$ y el cambio de tasa de respuestas a que esto conlleva, por una parte, y la presencia o ausencia de un desplazamiento de pico. La $P_{S 1}(R)$ se mantiene constante. Las posibilidades que se obtienen manteniendo constante $P_{S 1}(R)$, variando $P_{S 2}$ (R) y considerando los cambios en la tasa de respuesta $T$, ante $S 2$ se encuentran esquematizadas en la tabla 1.

De acuerdo con lo anterior el cuadro número 2 debe leerse así: "cuando $P_{s 2}(R)$ se mantiene constante y T aumenta"; el 6 se leería:"cuando $P_{S 2}(R)$ aumenta y T disminuye".

Los casos, 1, 3, 5, 6, 7 y 9 han sido estudiados por muchos investigadores (Dysart, Marx, Mclean, \& Nelson, 1974; Guttman, 1959; Huff, Sherman, \& Cohn, 1975; Mariner \& Thomas, 1973; Terrace, 1968; Wheatley \& Thomas, 1974; Wilkle, 1972; Yorczower, Dickson \& Gollub, 1966; Yarczower, Gollub \& Dickson, 1968). Los casos 2, 4 y 8 hasta el momento parecen no haber sido estudiados. Los tres experimentos que presentamos en este trabajo tienen como objetivo estudiar cada uno estos tres casos.

\section{TABLA 1}

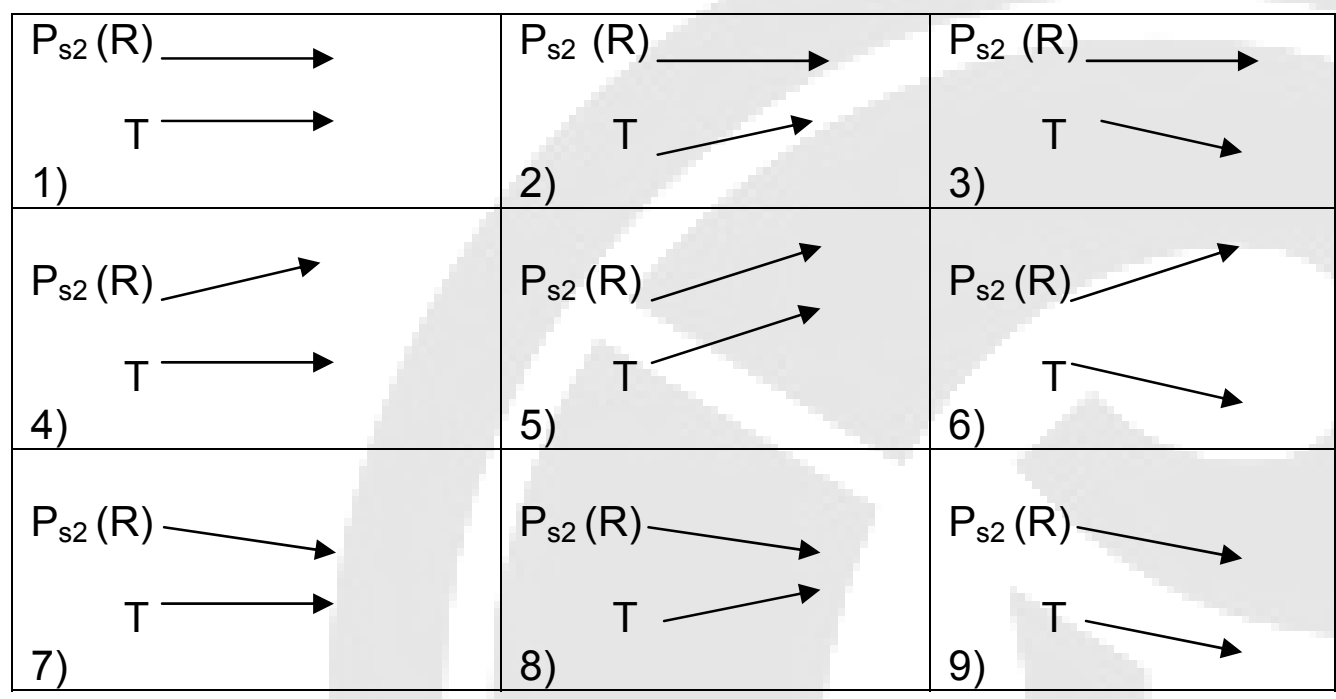

Las flechas deben leerse de la siguiente manera: no hay cambio; hay aumento; hay decremento. 


\section{Experimento 1}

En este experimento intentamos estudiar el caso 2, o sea, que se pretende mantener constante la probabilidad de refuerzo y se observa un aumento en la tasa de respuestas en el segundo componente. Para mayor comodidad aceptamos las siguientes convenciones: hablaremos de un múltiple $A$ cuyos componentes los denominamos $1_{\mathrm{A}} \vee 2_{\mathrm{A}}$ , y de un múltiple $B$ cuyos componentes los denominamos $1_{B} Y 2_{B}$. Para que se cumpla lo que se indica en el cuadro 2 tendríamos que lograr que:

$P_{S 2}(R)_{A}=P_{S 2}(R)_{B}$ y $T_{A}<T_{B}$

siendo $P_{S 2}(R)_{A}$ la probabilidad de refuerzo de la respuesta $R$ dado que el estímulo $S_{2}$ está presente durante el múltiple $A$ y $P_{S_{2}}(R)_{B}$ la probabilidad correspondiente durante el múltiple $B ; T_{A}$ y $T_{B}$ son las tasas de respuesta ante el estímulo $S_{2}$ en el múltiple $A$ y el múltiple $B$, respectivamente.

\section{Método}

\section{Sujetos}

Tres ratas albinas rattus norvergicus machos de 150 días de edad, identificadas como $\mathrm{H}-21, \mathrm{H}-22$ y H-23 sin experiencia previa en la caja de Skinner, privadas de agua durante 24 horas antes de cada sesión. Las ratas tendrán libre acceso al agua después de cada sesión durante un período de media hora.

\section{Aparatos}

La cámara experimental es una caja estándar para ratas (Ver Skinner, 1938) aislada totalmente de ruidos, marca Technical Service inc, de la B.R.S./L.V.E. provista de una palanca retráctil y un dispensador de agua automático. La programación de los múltiples se hará mediante circuitos electromecánicos. Los estímulos $S_{1} S_{2}$ serán tonos de 1000 cps. y 950 cps, respectivamente, producidos por un generador de audio de la Tech Serv. Los datos serán tomados automáticamente por un registro digital con posibilidad de impresión para cinco entradas paralelas (P.O.C. 505).

\section{Procedimiento}

Inicialmente las ratas serán entrenadas para tomar el líquido del dispensador de agua durante 3 sesiones. Un ruido producido por el dispensador servirá como señal de que el reforzador está disponible. Durante estas 3 sesiones la palanca no estará disponible. Se utilizará un programa de TF 15. Luego se llevarán a cabo 4 sesiones de moldeamiento; en la primera sesión se hará un período de calentamiento durante el cual se darán 5 refuerzos sin ninguna respuesta de presión a la palanca. Después de este período se reforzará solamente la respuesta de presionar la palanca, bajo un programa de refuerzo continuo (RFC) durante el cual se presentarán los estímulos $S_{1}$ y $S_{2}$ en sucesión semialeatoria; se permitirá un máximo de tres repeticiones sucesivas de cada estímulo. El número de reforzadores será de 30 y se mantendrá constante a través de todo el período de entrenamiento. La duración de cada presentación será de un minuto. Al final de cada minuto la luz de la cámara se apagará por 5 segundos (Black Out) con el fin de cambiar el valor de los estímulos de entrenamiento y también los estímulos de prueba durante la prueba de generalización. La palanca se retirará durante todos los B.O. (Black Out). 


\section{Entrenamiento no diferencial (o Múltiple A según lo convenido)}

Si $S_{1}$ y $S_{2}$ serán asociados cada uno a un IV2: tendremos entonces un Mult (IV2'-IV2'). Este programa estará en vigencia hasta el momento en que se cumpla lo siguiente: dado un intervalo cualquiera de un minuto, la tasa local asociada a este intervalo no debe diferir en más de un $10 \%$ de la tasa local asociada al intervalo de un minuto que siga al escogido. Este $10 \%$ de variabilidad tolerada no impedirá observar los cambios en la tasa ante $\mathrm{S}_{2}$.

\section{Entrenamiento diferencial (o Múltiple B según lo convenido)}

Después del entrenamiento no diferencial, las ratas serán expuestas a un programa Mult (IV2') tantas sesiones como sean necesarias para alcanzar el mismo criterio de estabilidad citado en el párrafo anterior en cada uno de los componentes del múltiple $\mathrm{S}_{1}$ será asociado al IV2' y $\mathrm{S}_{2}$ al Tand IV2'. DRH 0.5”.

Prueba de generalización: Después de completar el entrenamiento en discriminación cada sujeto recibirá una prueba de generalización en extinción, en la cual se presentarán once estímulos cuyas frecuencias serán $200,300, \ldots, 1.000,1.100$ y 1.200 cps. La duración de cada uno de los estímulos de prueba será de un minuto, con 5 segundos de B.O. entre cada presentación, presentando todos los estímulos en seis series diferentes al azar. La sesión será iniciada con $10^{\circ}$, de calentamiento bajo el programa en vigencia durante el entrenamiento diferencial.

\section{Resultados}

Se tomarán los siguientes datos. Tasa de respuesta para cada minuto de presentación de un estímulo en todas las tres etapas del experimento. Tasa global de respuestas para cada componente del múltiple en las dos primeras etapas. Para la tercera etapa, la prueba de generalización, las tasas locales para cada estimulo se presentarán en forma de gradientes de generalización absolutos y relativos. También se presentará un gradiente graficado a partir del número total de respuestas dado ante cada estímulo durante las seis presentaciones de cada uno de ellos.

\section{Experimento II}

En este experimento el paso del múltiple $A$ al B intenta establecer que:

$\mathrm{P}_{\mathrm{S} 2}(\mathrm{R})_{\mathrm{A}}<\mathrm{P}_{\mathrm{S} 2}(\mathrm{R})_{\mathrm{B}} \mathrm{Y} \mathrm{T}_{\mathrm{A}}=\mathrm{T}_{\mathrm{B}}$

Es decir, que intentaremos lograr que haya un aumento en la probabilidad de refuerzo sin que haya un aumento en la tasa de respuesta para el componente $2_{\mathrm{B}}$.

\section{Método}

\section{Sujetos}

Serán tres ratas de características idénticas al experimento anterior y se identificarán como $\mathrm{H}-24, \mathrm{H}-25$ y $\mathrm{H}-26$. 


\section{Aparatos}

Serán los mismos que se utilizan en el experimento anterior.

\section{Procedimiento}

El procedimiento previo al entrenamiento no-diferencial será igual al que se utilizará en el Experimento I.

\section{Entrenamiento no diferencial:}

Será el mismo utilizado en el Experimento I, con la excepción de que se empleará un programa múltiple IV1'-IV1'.

Entrenamiento diferencial: Después del entrenamiento no diferencial las ratas serán sometidas a un programa Mult (IV1') tomando " $x$ " como el promedio de los IRTs obtenidos en la ejecución del Mult IV1'-IV1'. Aquí el alternativo lo tenemos como aquel programa en el cual se refuerza la respuesta cada vez que se cumple uno de los dos requisitos: el exigido por DRL - $x$ o el exigido por IV1,. $S_{2}$ será el estímulo asociado con el Alt DEL-xIV1'.

Prueba de generalización. Se procederá en forma idéntica al experimento anterior.

\section{Resultados}

Se presentarán de igual forma que en el Experimento I.

\section{Experimento III}

Este último experimento fue diseñado para lograr que:

$$
P_{S 2}(R)_{A}>P_{S 2}(R)_{B} T_{A}<T_{B}
$$

Esto quiere decir que la probabilidad de refuerzo disminuye y la tasa de respuesta aumenta en el componente $2 \mathrm{~B}$.

\section{Método}

\section{Sujetos}

Serán también tres ratas de idénticas características a las anteriores identificadas como $\mathrm{H}-27, \mathrm{H}-28$ y $\mathrm{H}-29$.

\section{Aparatos}

Los aparatos serán los mismos utilizados en los Experimentos I y II.

\section{Procedimiento}

El procedimiento previo al entrenamiento no-diferencial será igual al utilizado en los experimentos I y II. 
Entrenamiento no-diferencial. Durante esta etapa estará en vigencia un Mult.

Entrenamiento diferencial. Durante esta etapa el programa en vigencia será Mult. Como siempre $\mathrm{S}_{2}$ estará asociado con el componente del múltiple que cambia de A a B.

Prueba de generalización. Igual a los Experimentos ly II.

\section{Resultados}

Serán presentados en la misma forma que en los experimentos I y II.

Hernán Escobedo D. 


\section{REFERENCIAS}

Dysart, J., Marx, M.H., McLean, J., \& Nelson, JA. "Peck shift as a function of multiple achedules of reinforcement". Journal of the Experimental Analysis ob Behavior, $1974,22,463-470$.

Eckernman, C.O. "Probability of reinforcement and the deveínpment of stimulus control". Journal of the Experimental Analysis of Behavior, 1969, 12, 551-559.

Guttman, N. "Generalization gradiente oround stimuli associated with different reinforcement schedules”. Journal of Experimental Psychology, 1959, 58, 325-340.

Huff, R.G., Sherman, J.E., \& Cohn, M. Sorne effects of response independent reinforcement on auditory generalization gradiente". Journal of the Experimental Analysis of Behavior. 1975, 23, 81-86.

Mariner, R. W., \& Thomas, D.R. "Reinforcement duration and the peck shift in postdiscrimination gradiente". Journal of the Experimental Analysis of Behavior 1969, 12, 759-766.

Terrace, H.S. "Discrimination learning, the peak shift, and behavioral contrast". Journal of the Experimental Analysis of Behavior, 1968, 11, 727-741.

Wheatley, K.L., \& Thomas, D.. "Relative and absolute density of reinforcement as factors influencing the peck shift". Journal of the Experi. mental Analysis of Behavior, 1974, 22, 409-418.

Wilkie, D.M. "The peck shift and behavioral contrast: Effects of discrimination training with delayed reinforcement”. Psychonomic science, 1972, 26, 257-258.

Yarczower M., Dickson, J.F., \& Gollub, L.R. "Sorne effects on géneralization gradiente of tandem schedules". Journal of the Experimental Analysis of Behavior, 1966, 9, 631639.

Yerczower, M., Gollub, L.R., \& Dickson. J.F. "Sorne effects of discriminative training with equated frequency of reinforcement". Journal of the Experimental Analysis of Behavior, 1968,11, 415-423. 